\title{
A new antigen on the epithelial membrane: its immunoperoxidase localisation in normal and neoplastic tissue
}

\author{
EADIE HEYDERMAN', KATE STEELE ${ }^{2}$, AND MICHAEL G. ORMEROD 2 \\ From the 'Unit of Human Cancer Biology, London Branch, Ludwig Institute for Cancer Research, Royal \\ Marsden Hospital, and the ${ }^{2}$ Institute of Cancer Research, Haddow Laboratories, Clifton Avenue, Sutton, \\ Surrey SM2 5PX, UK
}

SUMMARY Using antisera raised against defatted human cream we have demonstrated a new antigen on the epithelial membrane. An indirect immunoperoxidase technique on routinely processed histological sections was used, and we describe results which show that this antigen has a widespread but highly selective distribution, being apparently localised to membranes that have a secretory function. The antigen is carried by a variety of adenocarcinomas, suggesting that its localisation may have a role in the diagnosis and differential diagnosis of malignant disease.

The breast is a highly differentiated organ, and it seems likely that the surface of the mammary epithelium carries unique determinants. An antiserum to such a determinant might be of considerable value in the diagnosis and differential diagnosis of malignant disease. It could also elucidate aspects of cell differentiation in vitro as well as in vivo, and it might add a functional dimension to the present purely morphological classification of breast disease. The only convincing evidence for a tissuespecific antigen on the mammary membrane comes from the work of Ceriani et al. (1977), who describe an antiserum to defatted human cream, which is apparently specific to mammary epithelium, although comprehensive immunocytochemical experiments have yet to be published.

We have been raising antisera to elements of the human breast. Some of these sera define an antigen on the mammary membrane which, while it is not specific to breast, does show specificity for secretory epithelia. In this paper we describe a study of the distribution of this epithelial membrane antigen using immunoperoxidase staining of histological sections.

\section{Material and methods}

\section{ANTISERA}

While several antisera obtained by injecting rabbits

Received for publication 3 July 1978 with material from hepatic metastases of mammary carcinomas reacted with the epithelial membrane, the most effective were obtained using defatted cream (Ceriani et ul., 1977), prepared for us by Mr A. Imam by extracting washed cream twice with two volumes of chloroform and twice with one volume of ether. This yielded membranes of the fat globules, which are a convenient antigen since they aie formed from the plasma membrane of breast epithelial cells (Soake and Heald, 1974). Membranes were suspended in water and an emulsion was prepared with complete Freund's adjuvant. Rabbits were injected subcutaneously at monthly intervals alternately in the nuchal area and in the rump. Antisera of high titre, as judged by immunoperoxidase staining, were obtained two weeks after the fourth injection.

The antisera were absorbed with plasma, extracts of kidney and liver (made by extraction of tissue with $3 \mathrm{~mol} / \mathrm{l} \mathrm{KCl}$ ), non-specific cross-reacting antigen (Von Kleist et al., 1972), and lactoferrin. The absorbents were immobilised by reaction with cyanogen bromide-activated Sepharose so that any antigen-antibody complexes formed could be removed from the serum by centrifugation.

Sheep anti-rabbit- $\gamma$-globulin serum was prepared by injecting rabbit IgG with the same schedule as that used for rasing antisera in rabbits. Two weeks after the fourth injection the sheep was exsanguinated and the serum was harvested.

Antisera to carcinoembryonic antigen, $\alpha$-lactalbu- 
min, and ferritin were prepared in rabbits; those to lactoferrin, secretory piece of IgA, and muramidase were purchased from Dakopatts Ltd. Other antisera were generous gifts: normal cross-reacting antigen from Dr E. Engvall, $\beta$-oncofetal antigen from Dr J. P. Mach, casein from Dr R. Woods, pregnancy specific $\beta_{1}$-glycoprotein from $\mathrm{Dr} \mathrm{H}$. Bohn, and cystic disease fluid protein from $\mathrm{Dr}$ Haagensen.

\section{IMMUNOPEROXIDASE STAINING}

Our indirect immunoperoxidase technique, including our method for blocking endogenous peroxidase, has been described in detail elsewhere (Heyderman and Neville, 1976; Heyderman, 1978). Specific sheep anti-rabbit- $\gamma$-globulin antibodies were purified by affinity chromatography, and peroxidase conjugates were prepared by a modification of Nakane's method (Nakane and Kawaoi, 1974; Heyderman, 1978). To ensure uniformity, each batch of conjugate was tested for optimum working dilution and stored frozen, diluted in aliquots until required.

We have established that the most valid negative immunocytochemical control is the complete extinction of staining when the antiserum is absorbed with the relevant antigen. In this case, however, since the antigen has not been characterised and purified, we absorbed the antiserum with a fraction prepared from human milk (see below). To check whether the abolition of staining was non-specific, we used this fraction to absorb inappropriate antisera. The staining of malignant syncytiotrophoblast with antihuman chorionic gonadotrophin and of carcinoma of the colon with anti-carcinoembryonic antigen was unaffected.

\section{TISSUES}

The sections were cut at $3-5 \mu \mathrm{m}$ from mainly formalin-fixed, routinely prepared, paraffin-embedded blocks. Most of the material was from surgical biopsies while some was obtained at necropsy; sections of marrow prepared from bone trephines were decalcified in dilute nitric acid. Cells in tissue culture were grown in monolayers; the cells were harvested either mechanically or by treatment with trypsin and a pellet fixed for one hour in Bouin's solution (our preferred fixative for immunocytochemistry). Alternatively, cells were grown on coverslips and fixed as monolayers in Bouin's solution for five minutes.

PREPARATION OF ACTIVE FRACTION FROM HUMAN MILK

We found that if the antisera were absorbed with human milk, the immunoperoxidase staining was abolished. Using this as a test system, we are trying to isolate soluble epithelial membrane antigen from milk; this work is still at a preliminary state. In order to absorb antisera for controls, we used a partially purified fraction of epithelial membrane antigen from milk.

Skimmed human milk was centrifuged at $4 \times 10^{4}$ $\mathrm{rev} / \mathrm{min}\left(10^{5} \mathrm{~g}\right)$ for five hours to remove fragments of membranes and casein micelles. Ammonium sulphate was added to the supernatant to $40 \%$ saturation, the resulting precipitate was discarded, and the solution was brought up to $60 \%$ saturation. The precipitate was collected by centrifugation, dissolved in water, dialysed against $0.1 \mathrm{~mol} / 1$ phosphate, $0.25 \mathrm{~mol} / \mathrm{l}$ $\mathrm{NaCl}, 1 \mathrm{~m} \mathrm{~mol} / 1 \mathrm{EDTA}, \mathrm{pH} 8 \cdot 0$, and applied to a column of Sepharose 6B. The active fraction eluted from the column with a molecular weight of about $10^{6}$ daltons was dialysed against $0.01 \mathrm{~mol} / 1 \mathrm{tris} / \mathrm{HCl}$, $0.1 \mathrm{~mol} / 1 \mathrm{NaCl}, \mathrm{pH} 8.5$, and applied to a column of DEAE Sephadex (A50) in this buffer. The fraction eluted in the same buffer contained epithelial membrane antigen and was used to absorb antisera.

\section{Results}

BREAST

On an immunodiffusion plate the antisera raised against defatted cream demonstrated a multiplicity of precipitin lines against milk and different extracts of the milk fat globule membrane or cream. After absorption with plasma, extracts of kidney and liver, normal cross-reacting antigen, and lactoferrin, precipitin lines could no longer be detected by immunodiffusion. In contrast, sections of normal or malignant breast were still stained by the immunoperoxidase technique. The antisera reacted with all the mammary tissues studied-lactating, benign, and malignant. The most striking feature was the localisation of staining on the surface of the luminal membrane (see Fig. 1). It is this that leads us to conclude that the antisera detect an epithelial membrane antigen.

In moderately or well differentiated mammary carcinomas the antigen was mainly concentrated on the luminal border of the malignant acini (Fig. 2). In poorly differentiated adenocarcinomas the antigen was also found in the cytoplasm; a similar finding has been reported for the colonic membrane antigen, carcinoembryonic antigen (Heyderman and Neville, 1976; Pascal et al., 1977).

The presence of epithelial membrane antigen in the cytoplasm allowed the observation of mammary carcinoma cells infiltrating bone marrow; in larger clumps the luminal membranes of small acini were picked out (Fig. 3). A xenograft of a primary breast carcinoma in nude mice (prepared for us by Ms S. I. Detre) continued to express epithelial membrane 

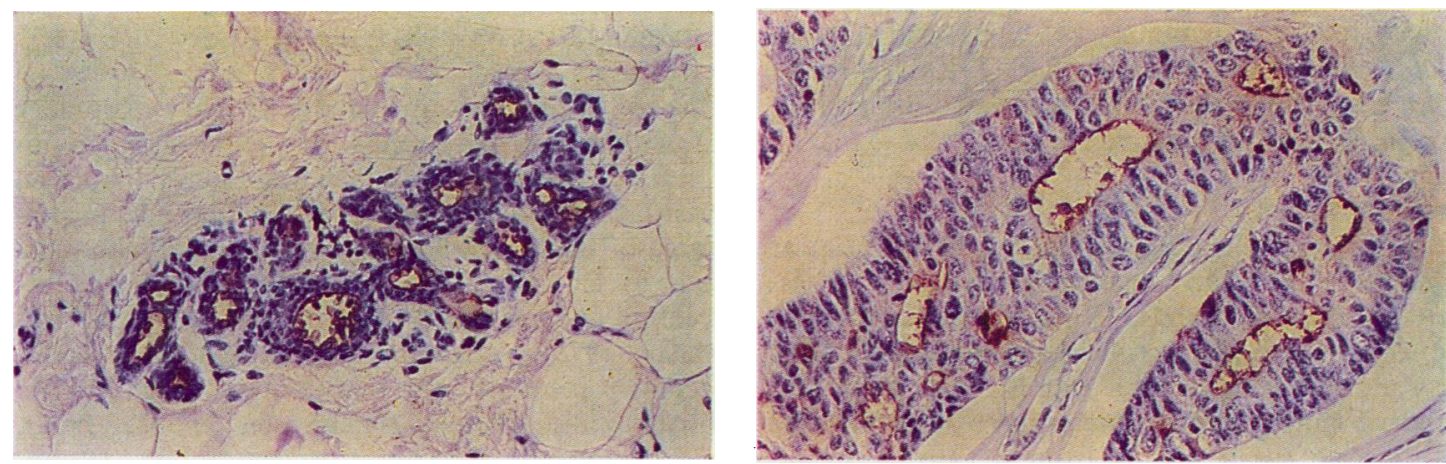

FIG. 1

FIG. 2
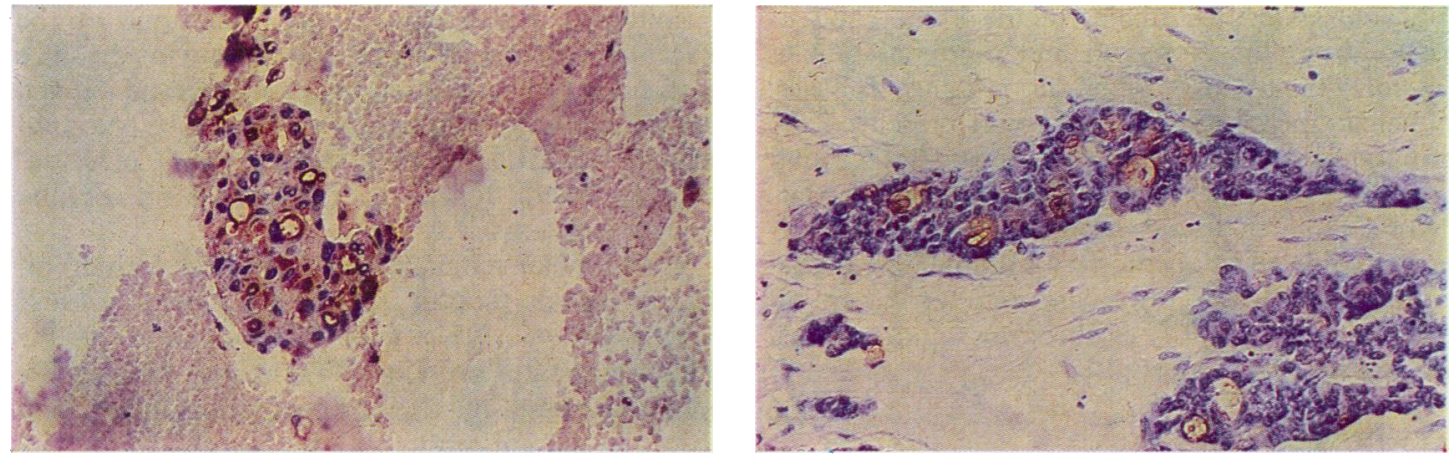

FIG. 3

FIG. 4

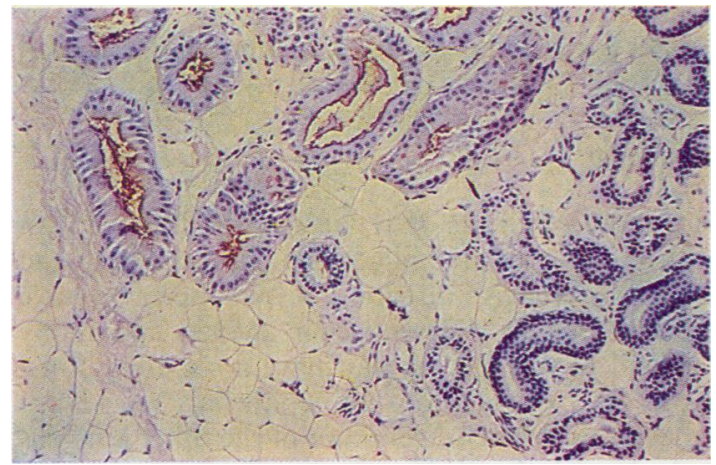

FIG. 5

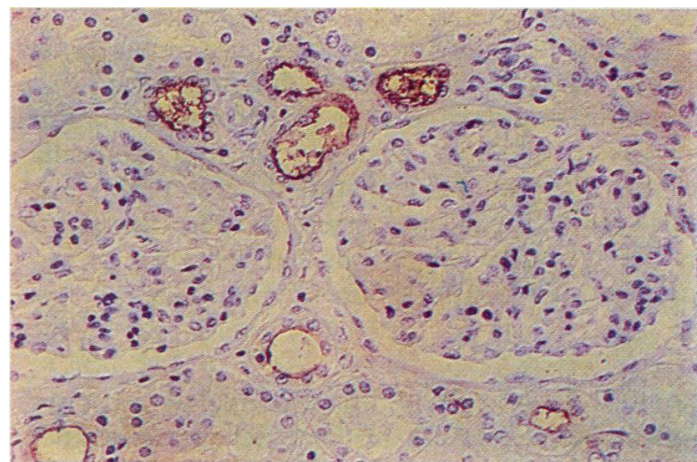

FIG. 6

Figs 1-6 Indirect immunoperoxidase stain with antiserum to epithelial membrane antigen diluted 1:50-1:200, depending on titre of bleed. Meyer's haemalum counterstain.

Fig. 1 Normal breast lobule showing staining of luminal membrane and secretions. Myoepithelial cells, fat, and stroma are unstained $(\times 160)$.

Fig. 2 Infiltrating ductal carcinoma of breast. The staining is mainly confined to the luminal membrane of the malignant acini but there is a little cytoplasmic staining. The stroma is again completely negative $(\times 160)$.

Fig. 3 Marrow aspirate from patient with metastatic breast carcinoma. The clump of malignant cells shows both membrane and cytoplasmic positivity. (The faint brown tinge of the erythrocytes is due to fixation in Zenker's solution $)(\times 160)$.

Fig. 4 Adenocarcinoma of lung. The stuining is mainly in microacini and their contained necrotic debris $(\times 160)$.

Fig. 5 Hyperhidrosis. In this axillary biopsy the luminal membrane of the apocrine glands is positive, while the ducts are negative. Elsewhere in the section luminal staining of the eccrine sweat glands, but not their ducts, is present $(\times 64)$.

Fig. 6 Residual normal kidney lateral to a hypernephroma. Glomeruli and proximal tubules are negative, while distal tubules show positive luminal membranes $(\times 160)$. 
antigen, as did a mammary cell line, MCF7 (Soule et al. (1973) grown for us by Dr D. Easty). In the latter case, the antigen was distributed abundantly though unevenly on the membrane.

\section{OTHER ORGANS}

Most organs showing glandular differentiation contained epithelial membrane antigen. These included salivary glands, pancreas, stomach, some bronchial mucus glands, bile ducts, endometrial and cervical glands, decidual glands, and sebaceous glands. Figure 5 shows immunoperoxidase staining of apocrine sweat glands from a case of hyperhidrosis. The glands show luminal staining while the eccrine ducts were virtually negative. Most intriguing was the presence of epithelial membrane antigen on distal and collecting tubules of the kidney and its absence from the glomeruli and proximal tubules (Fig. 6). It was also found in pituitary follicles. Epithelial membrane antigen was absent from normal colon and ileum and from the cellular elements of normal marrow and lymphoid tissue. It was sometimes found, however, in histologically normal colon in association with tumour or inflammation. Epididymis, testis, and rete testis were all negative. Staining of erythrocytes and stromal elements was not observed.

\section{MALIGNANT TUMOURS}

Epithelial membrane antigen has been demonstrated in adenocarcinomas from a wide variety of sites, including stomach, colon, prostate, uterus, ovary, lung (Fig. 4) pleura, and thyroid. The material was found in hypernephromas and in those nephroblastomas showing evidence of tubular differentiation. In a malignant teratoma it was found as a luminal component of those elements showing glandular but not those showing neural differentiation even when rosettes with central lumina were formed. It was not detected on or in oat cell carcinomas, seminomas, or non-Hodgkin's lymphomas.

\section{FETAL TISSUE}

In a limited study of fetal tissue, epithelial membrane antigen was observed in fetal sebaceous glands, pulmonary alveolae, on the most superficial layers of the bladder, and possibly in the colon though not in the ileum. A thin layer of the antigen appeared to be present on the microvilli of the fetal syncytiotrophoblast.

OTHER MAMMALS

Lactating mouse mammary tissue, lung, liver, kidney, and spleen were all negative as were the mouse tissues surrounding positive human tumour xenografts.

\section{COMPARISON WITH OTHER KNOWN} ANTIGENS

Our control sections included a primary mammary carcinoma and its lymph node metastasis, an infiltrating carcinoma in a lactating, partly involuted breast, and a moderately differentiated adenocarcinoma of the colon. Sections were stained with antisera to carcinoembryonic antigen, normal crossreacting antigen, $\beta$-oncofetal antigen (Fritsché and Mach, 1975), $\alpha$-lactalbumin, ferritin, lactoferrin, secretory piece of $\operatorname{IgA}$, muramidase, casein, cystic disease fluid protein (Haagensen et al., 1977),

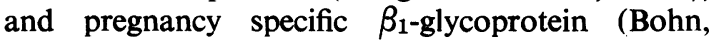
1971). In no case did the distribution of staining in all three blocks resemble the pattern achieved with the antisera to epithelial membrane antigen.

Epithelial membrane antigen is not one of the placental proteins we have studied previously since in sections of placenta, epithelial membrane antigen was located on villous membranes while the placental proteins, $\beta$-HCG, $\beta$-SPI, and human placental lactogen, were found in the cytoplasm of the syncytiotrophoblast.

Most tubular renal antigens have been located on the proximal tubules (Edgington et al., 1967; Naruse et al., 1973) with the exception of the Tamm-Horsfall protein (Friedman, 1966). We piepared some of this protein from urine and absorbed the antiserum with it. No loss of activity resulted, showing lack of identity with epithelial membrane antigen. A similar result was obtained when we absorbed antiserum with either of the membrane antigens, carcinoembryonic antigen or normal cross-reacting antigen, confirming the lack of identity of epithelial membrane antigen with them.

\section{Discussion}

Our results indicate that we have antisera to an epithelial membrane antigen that has a widespread but highly selective distribution. We appear to have identified a new differentiation antigen apparently localised to membranes that have a secretory function. The distribution of epithelial membrane antigen was different from that of the other antigens studied, and the difference was confirmed in the case of carcinoembryonic antigen, normal crossreacting antigen, and ferritin by absorption of the antiserum. The absence of staining of erythrocytes showed that it was unlikely to be a blood group antigen.

The only similar surface differentiation antigen reported is that of Ceriani et al. (1977) but they concluded, from an immunofluorescence study of cell lines, that their antiserum was specific for breast. It is possible that we are both studying the same 
antigen and that the differences in specificity are due to differences in the method of study. This question will be resolved by an exchange of reagents, which is being arranged.

We have assumed that, after absorption, our antisera can detect only one component on the epithelial membrane. Our assumption is probably justified since a fraction from milk-containing partially purified epithelial membrane antigen removed all remaining activity from the antisera (see Material and methods). Conclusive proof will be supplied only when the antigen has been isolated and characterised.

Purification of epithelial membrane antigen is clearly a priority. While human milk offers a convenient source, our early results indicate that the physical properties of the soluble antigen depend on the treatment of the sample of milk.The yield appears to be rather low, and we might have to use an alternative source of material-possibly a large metastatic deposit of mammary carcinoma obtained at necropsy.

We speculate that the antigen has a specific function related directly or indirectly to the secretory process. When we have succeeded in isolating the molecule, a detailed characterisation might yield clues as to its role, although this is far from assured.

Our data on the immunoperoxidase localisation of epithelial membrane antigen already suggest a role for these antisera in the diagnosis and differential diagnosis of malignant disease. Several such aspects are under active investigation.

We thank Professor A. M. Neville for most valuable support and guidance, Ms P. Davies for excellent technical assistance, and the pathologists who generously gave us access to their histological material.

\section{References}

Bohn, H. (1971). Nachweis und Charakterisierung von Schwangerschafts-proteinen in der menschlichen Placenta, sowie ihre quantitative immunologische Bestimmung im Serum schwangerer Frauen. Archiv für Gynaekologie, 210, 440-457.

Ceriani, R. L., Thompson, K., Peterson, J. A., and Abraham, S. (1977). Surface differentiation antigens of human mammary epithelial cells carried on the human milk fat globule. Proceedings of the National Academy of Sciences of the United States of America, 74, 582-586.
Edgington, T. S., Glassock, R. J., Watson, J. I., and Dixon, F. J. (1967). Characterisation and isolation of specific renal tubular epithelial antigens. Journal of Immunology, 99, 1199-1210.

Friedman, T. (1966). Immunofluorescent localisation of Tamm-Horsfall mucoprotein. Experientia, 22, 624-625.

Fritsché, R., and Mach, J. P. (1975). Identification of a new oncofoetal antigen associated with several types of human carcinomas. Nature, 258, 734-737.

Haagensen, D. E., Jr., Mazoujian, G., Holder, W. D., Jr., Kister, S. J., and Wells, S. A., Jr. (1977). Evaluation of a breast cyst fluid protein detectable in the plasma of breast carcinoma patients. Annals of Surgery, 185, 279-285.

Heyderman, E. (1978). Multiple tissue markers in human malignant testicular tumours. 5th Meeting of the International Research Group for Carcinoembryonic Proteins, Copenhagen. Scandinavian Journal of Immunology, 7/2, Supplement 8. (In press.)

Heyderman, E., and Neville, A. M. (1976). A shorter immunoperoxidase technique for the demonstration of carcinoembryonic antigen and other cell products. Journal of Clinical Pathology, 30, 138-140.

Nakane, P. K., and Kawaoi, A. (1974). Peroxidaselabeled antibody: a new method of conjugation. Journal of Histochemistry and Cytochemistry, 22, 1084-1091.

Naruse, T., Kitamura, K., Miyakawa, Y., and Shibita, S. (1973). Deposition of renal tubular epithelial antigen along the glomerular capillary walls of patients with membranous glomerulonephritis. Journal of Immunology, 110, 1163-1166.

Pascal, R. R., Mesa-Tejada, R., Bennett, S. J., Garces, A., and Fenoglio, C. M. (1977). Carcinoembryonic antigen. Archives of Pathology and Laboratory Medicines, 101, 568-571.

Soake, R. G., and Heald, C. W. (1974). In Lactation, edited by B. L. Lasson and V. R. Smith, Volume 2, pp. 147-187. Academic Press, New York.

Soule, H. D., Vazquez, J., Long, A., Albert, S., and Brennan, M. (1973). A human cell line from a pleural effusion derived from a breast carcinoma. Journal of the National Cancer Institute, 51, 1409-1413.

Von Kleist, S., Chavanel, G., and Burtin, P. (1972). Identification of an antigen from normal human tissue that cross-reacts with the carcinoembryonic antigen. Proceedings of the National Academy of Sciences of the United States of America, 69, 24922494.

Requests for reprints to: Dr E. Heyderman, Unit of Human Cancer Biology, Ludwig Institute for Cancer Research, The Haddow Laboratories, Clifton Avenue, Sutton, Surrey SM2 5PX,UK 